\title{
Acute pulmonary edema due to occult air embolism detected on an automated anesthesia record: illustrative case
}

\author{
Samuel Wood, MD, and Gennadiy Fuzaylov, MD \\ Department of Anesthesia, Critical Care, and Pain Medicine, Massachusetts General Hospital, Boston, Massachusetts
}

\begin{abstract}
BACKGROUND The authors report a case of venous air embolism (VAE) during a pediatric posterior fossa craniotomy with resulting pulmonary edema requiring postoperative ventilation. Pulmonary edema is a known but rare complication of VAE, and diagnosis and treatment are discussed.

OBSERVATIONS The embolism was undetected during the surgical procedure, and the first clinical sign of respiratory decompensation appeared an hour after the initial insult, with imaging suggesting acute pulmonary edema. A transient but significant end-tidal carbon dioxide decrease was detected on postoperative review of the anesthesiology record.
\end{abstract}

LESSONS This report highlights an uncommon sequela of VAE and the importance of post hoc automated record review for intraoperative event analysis.

https://thejns.org/doi/abs/10.3171/CASE2075

KEYWORDS air embolism; pulmonary edema; automated record; posterior fossa

Venous air embolism (VAE) is typically associated with posterior fossa surgeries. Incidence varies widely by method of detection and precipitating surgery, with mortality rates as high as $21 \% .{ }^{1}$ There is likely a proportion of subclinical VAE that goes undetected. ${ }^{2}$ Clinical severity is thought to be correlated with the size of the embolus and the speed at which the air was entrained. Lethal volumes are estimated to be $3-5 \mathrm{ml} / \mathrm{kg}$. Less severe embolisms have a broad range of cardiopulmonary sequelae. One rare but important sequela is postembolism noncardiogenic edema. ${ }^{2}$ We discuss a case of pulmonary edema following occult air embolism during posterior fossa craniotomy and the role of the anesthesia record in its detection.

\section{Illustrative Case}

A 6-year-old female with a history of Chiari 1 malformation, prothrombin mutation, and laryngeal cleft status post repair presented with several months of exertional occipital headaches. Magnetic resonance imaging of the brain revealed decreased cerebrospinal fluid at the cervicomedullary junction, tonsillar descent to C1-2, and medullary kink. She was taken to the operating room at our teaching institution for posterior fossa craniotomy, decompression, C1 laminectomy, and duraplasty.

General anesthesia was induced with a volatile agent, and the patient was intubated without issue. Anesthesia was maintained with a combination of volatile anesthetic, propofol, and remifentanil infusions. The patient was positioned prone with her head in pins below the level of the heart. Fentanyl, morphine, acetaminophen, and ketorolac were administered during the case for analgesia. The patient received a total of $30 \mathrm{ml} / \mathrm{kg}$ of lactated ringers. Intraoperative monitoring included neuromonitoring (motor evoked potentials, sensory evoked potentials, and electroencephalography), precordial Doppler, electrocardiogram, pulse oximetry, noninvasive blood pressure measuring, and end-tidal carbon dioxide.

Incision to closure time was 4 hours and 15 minutes. Until emergence and extubation, there were no aberrations of oxygen saturation, blood pressure, heart rate, or heart rhythm. There was a 1-minute period 3 hours into the case when end-tidal carbon dioxide dropped from 34 to 15 (Fig. 1). This transient decrease was only discovered after postoperative minute-byminute analysis of the anesthesia record. This change in end-tidal carbon dioxide resolved spontaneously. The remainder of the surgical course and neuromonitoring were unremarkable.

Upon emergence and extubation, the patient was almost immediately tachypneic to 50 respirations a minute. There was no evidence of upper airway obstruction or laryngospasm on examination. Her blood pressure and heart rate at the time of desaturation were $116 / 65 \mathrm{mmHg}$ of mercury and 103 beats per minute, respectively. Tachypnea was 


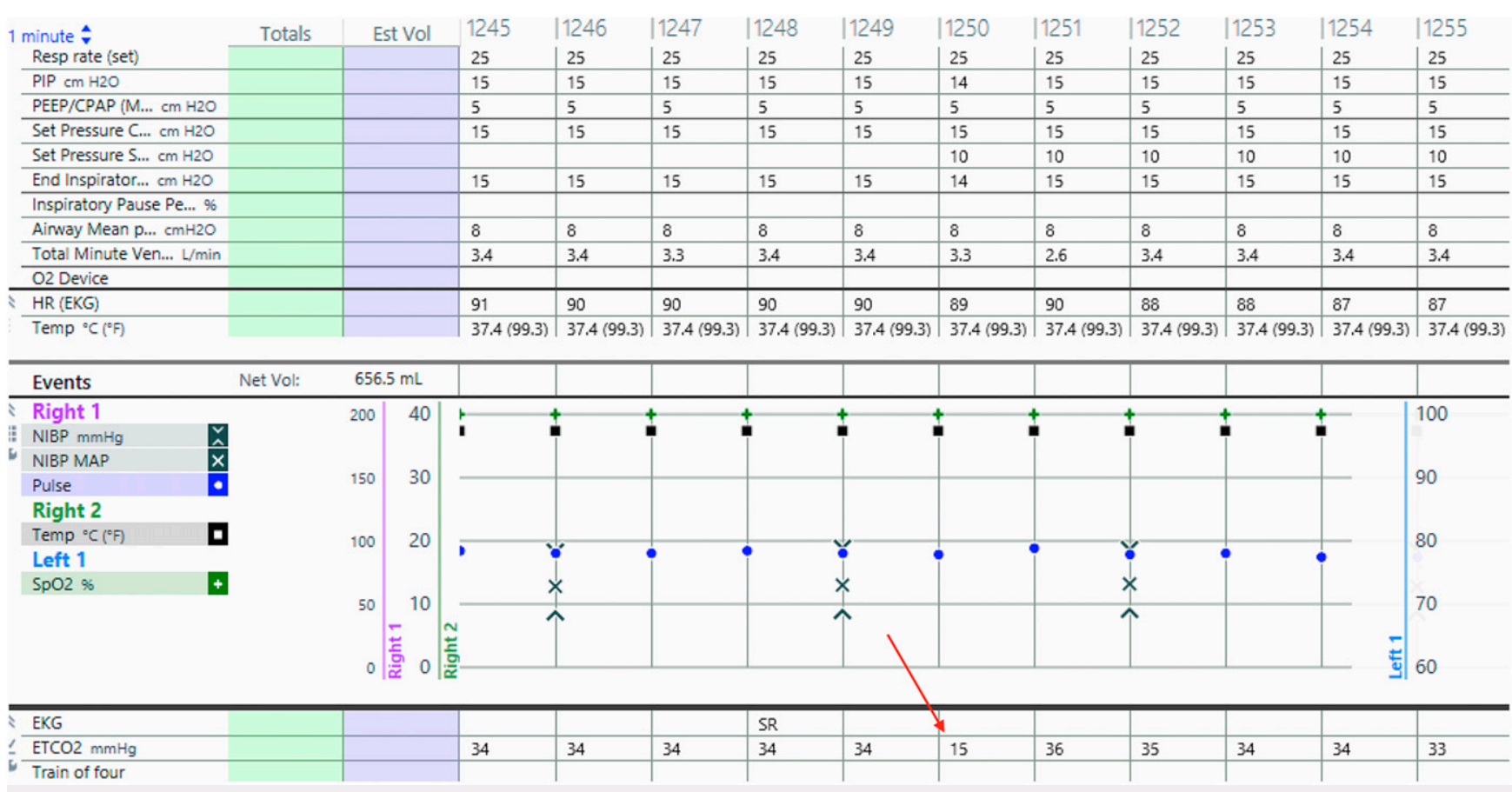

FIG. 1. Screenshot of the anesthesia record with arrow pointing to end-tidal carbon dioxide decrease.

interpreted as pain, and fentanyl $(0.5 \mu \mathrm{g} / \mathrm{kg})$ was administered with minimal change in the respiratory rate. The patient desaturated to $74 \%$ oxygen while transitioning to face mask with a flow of $6 \mathrm{l} / \mathrm{min}$. Her oxygen saturation improved to $95 \%$ with positive-pressure mask ventilation (positive end-expiratory pressure of $6 \mathrm{~cm}$ of water).

A chest radiograph was obtained in the operating room and showed marked pulmonary edema bilaterally. She was reanesthetized and a laryngeal mask airway was placed. Due to her prothrombin gene mutation, she underwent chest computed tomography (CT), which was negative for embolism but revealed diffuse ground-glass and dependent opacities consistent with edema and atelectasis (Fig. 2). She was then intubated and transferred to the pediatric intensive care unit. There she received further positive pressure ventilation and diuresis and was extubated on postoperative day 1. A repeat chest radiograph prior to extubation showed significantly improved edema. The patient was transferred to the floor and discharged on postoperative day 4 without further complication.

\section{Discussion}

\section{Observations}

The etiology of the patient's postextubation acute respiratory distress and pulmonary edema was initially unclear due to her intraoperative hemodynamic and respiratory stability. Post hoc analysis of the patient's record was only remarkable for the transient drop in endtidal carbon dioxide. We believe this end-tidal decrease in a high-risk surgical setting to be most consistent with VAE. This diagnosis was made possible by the automated anesthesia record, which is a useful tool for post hoc detection of intraoperative incidents. ${ }^{3}$ Automation of data collection and integration with the Epic electronic medical record allowed for minute-to-minute analysis of physiological, pharmacological, and ventilator variables.

VAE is a rare but morbid complication of surgery and invasive procedures. It is most commonly associated with open neurosurgical procedures, especially those in the posterior fossa. ${ }^{4}$ Common methods for detection of VAE include transesophageal echocardiography, precordial Doppler, end-tidal nitrogen, and end-tidal carbon dioxide. A combination of modalities is utilized in conjunction with clinical suspicion to make the diagnosis. ${ }^{5}$ In our case, a precordial Doppler was utilized because it is the most sensitive noninvasive monitor for VAE. However, the precordial Doppler is subject to inaccuracy from mispositioning (especially when the patient is prone) and interference from electrocautery, which may have prevented VAE detection in our case. ${ }^{6}$

If VAE is detected intraoperatively, its treatment is focused on the prevention of further entrainment, aspiration of air, and cardiopulmonary support. The extent of treatment is dictated by the respiratory and cardiopulmonary impact. To stop further accumulation of air, flood

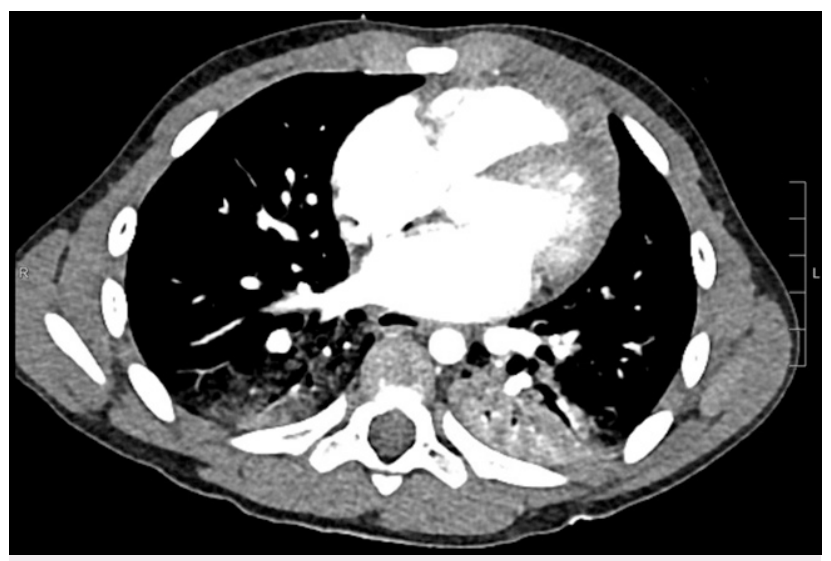

FIG. 2. Postoperative chest CT with bilateral pulmonary edema. 
the field with saline, compress the jugular veins, and reposition the head below the level of the heart. To contain entrapped air within the ventricular apex and avoid outflow obstruction, the patient can be put into the Trendelenburg and the left lateral decubitus positions. Air can be directly aspirated from the right heart with a central line if present. ${ }^{5}$

Our patient developed noncardiogenic pulmonary edema, an infrequent but reported complication of VAE. ${ }^{7}$ Case reports in the literature are varied with regard to the clinical presentation of the embolism prior to pulmonary edema. ${ }^{7-9}$ Almost all are associated with one or more transient decreases in end-tidal carbon dioxide with varying amounts of oxygen desaturation and hemodynamic instability. However, edema can occur after embolisms that do not cause hemodynamic instability. ${ }^{10}$ Just as with our patient, chest radiographs within several hours of the initial emboli show bilateral pulmonary edema in most cases. Proposed mechanisms for post-VAE edema include increased capillary hydrostatic pressure and damage to pulmonary capillaries, both from mechanical obstruction and oxygen metabolites. ${ }^{11}$ One study introduced microemboli into both normal and profoundly leukopenic sheep and found that postembolism microvascular permeability was significantly reduced in the leukopenic group. This finding suggests that there is a likely inflammatory or immune component to pulmonary edema from emboli. ${ }^{12}$

Our treatment of post-VAE pulmonary edema is similar to that of other noncardiogenic etiologies and focused on respiratory support with supplemental oxygen, positive pressure, and diuresis. The patient had significant clinical improvement and was extubated within 24 hours. Other cases noted meaningful recovery occurring in anywhere from several hours to several weeks. Post-VAE edema is predominately self-limited due to spontaneous resorption of the embolus. ${ }^{13}$

\section{Lessons}

Our case illustrates an uncommon presentation of VAE. We demonstrate that even hemodynamically insignificant embolisms can lead to clinically significant pulmonary edema in a pediatric patient. Because the patient was stable without significant respiratory compromise at the time of insult, it is difficult to say what could have been done differently in the moment with earlier recognition of the end-tidal carbon dioxide decrease. However, early identification could have raised awareness for possible future complications. Ultimately, focus should be placed on preoperative VAE risk stratification and prevention. Intraoperative detection of transient emboli can be difficult, and even with detection, further sequelae can occur. In this case VAE led to reintubation, intensive care unit admission, and a longer hospital stay.

Additionally, this case highlights the role of the automated anesthetic record in the detection and analysis of intraoperative incidents. If the end-tidal carbon dioxide decrease had not been detected and recorded, it is likely that the etiology of this patient's edema would have remained unclear. Rarely in a clinical setting are patient data recorded and stored with such frequency and detail. This should be utilized as a resource, both intra- and postoperatively, to facilitate clinical diagnosis and patient care.

\section{References}

1. McCarthy CJ, Behravesh S, Naidu SG, et al. Air embolism: diagnosis, clinical management and outcomes. Diagnostics (Basel) 2017;7(1):5.

2. Mirski MA, Lele AV, Fitzsimmons L, et al. Diagnosis and treatment of vascular air embolism. Anesthesiology. 2007;106(1):164-177.

3. Sanborn KV, Castro J, Kuroda M, et al. Detection of intraoperative incidents by electronic scanning of computerized anesthesia records. Comparison with voluntary reporting. Anesthesiology. 1996;85(5):977-987.

4. Palmon SC, Moore LE, Lundberg J, et al. Venous air embolism: a review. J Clin Anesth. 1997;9(3):251-257.

5. Shaikh N, Ummunisa F. Acute management of vascular air embolism. J Emerg Trauma Shock. 2009;2(3):180-185.

6. Chang J-L, Albin MS, Bunegin L, etal. Analysis and comparison of venous air embolism detection methods. Neurosurgery. 1980;7(2) 135-141.

7. Saigal D, Ganjoo P, Tetarway M, et al. Acute pulmonary edema and thrombocytopenia following venous air embolism during sitting position neurosurgery. Asian J Neurosurg. 2017;12(2):214-216.

8. Chandler WF, Dimcheff DG, Taren JA. Acute pulmonary edema following venous air embolism during a neurosurgical procedure. Case report. J Neurosurg. 1974;40(3):400-404.

9. Ishida K, Hishinuma M, Miyazawa M, et al. Pulmonary edema due to venous air embolism during craniotomy: a case report. Article in Japanese. Masui. 2008;57(10):1257-1260.

10. Frim $D M$, Wollman $L$, Evans $A B$, et al. Acute pulmonary edema after low-level air embolism during craniotomy. Case report. J Neurosurg. 1996;85(5):937-940.

11. El Kettani C, Badaoui R, Fikri M, et al. Pulmonary oedema after venous air embolism during craniotomy. Eur J Anaesthesiol. 2002; 19(11):846-848.

12. Flick MR, Perel A, Staub NC. Leukocytes are required for increased lung microvascular permeability after microembolization in sheep. Circ Res. 1981;48(3):344-351.

13. Kuhn M, Fitting J-W, Leuenberger P. Acute pulmonary edema caused by venous air embolism after removal of a subclavian catheter. Chest. 1987;92(2):364-365

\section{Disclosures}

The authors report no conflict of interest concerning the materials or methods used in this study or the findings specified in this paper.

\section{Author Contributions}

Conception and design: both authors. Acquisition of data: Wood. Analysis and interpretation of data: Wood. Drafting the article: Wood. Critically revising the article: both authors. Reviewed submitted version of manuscript: Wood. Approved the final version of the manuscript on behalf of both authors: Wood. Administrative/technical/ material support: Fuzaylov.

\section{Correspondence}

Samuel Wood: Massachusetts General Hospital, Boston, MA. swood13@partners.org. 